\title{
Exploring Potential Social Influences on Brain Potentials during Anticipation of Tactile Stimulation
}

\author{
Guannan Shen \\ Joni N. Saby \\ Ashley R. Drew \\ Peter J. Marshall
}




\begin{abstract}
This study explored interpersonal influences on electrophysiological responses during the anticipation of tactile stimulation. It is well-known that broad, negative-going potentials are present in the event-related potential (ERP) between a forewarning cue and a tactile stimulus. It has also been shown that the alpha-range mu rhythm shows a lateralized desynchronization over central electrode sites during anticipation of tactile stimulation of the hand. The current study used a tactile discrimination task in which a visual cue signaled that an upcoming stimulus would either be delivered 1500 ms later to the participant's hand, to a task partner's hand, or to neither person. For the condition in which participants anticipated the tactile stimulation to their own hand, a negative potential (contingent negative variation, CNV) was observed in the ERP at central sites in the 1000 milliseconds prior to the tactile stimulus. Significant mu rhythm desynchronization was also present in the same time window. The magnitudes of the ERPs and of the mu desynchronization were greater in the contralateral than in the ipsilateral hemisphere prior to right hand stimulation. Similar ERP and EEG changes were not present when the visual cue indicated that stimulation would be delivered to the task partner or to neither person. The absence of social influences during anticipation of tactile stimulation, and the relationship between the two brain signatures of anticipatory attention (CNV and mu rhythm) are discussed.
\end{abstract}

Key words: EEG, mu rhythm, somatosensory anticipation, CNV 


\section{Introduction}

Increasing efforts are being dedicated to studying the role of somatosensory processes in social perception (see Keysers et al., 2010; Zaki et al., 2016, for review), including the question of whether brain networks involved in somatosensory processing are active in response to observing tactile stimulation of others. Shared activations in primary (SI) and secondary somatosensory cortex (SII) during direct somatosensory stimulation and during observation of another person being touched have been reported in a number of studies employing functional magnetic resonance imaging (fMRl; Blakemore et al., 2005; Ebisch et al., 2008; Keysers et al., 2004; Kuehn et al., 2014; Schaefer et al., 2009), with additional activation in anterior cingulate cortex (ACC) and anterior insular $(\mathrm{Al})$ when experiencing or witnessing painful stimulation (Costantini, et al., 2008; Jackson et al., 2006; Lamm et al., 2009). In addition to these fMRI findings, there is a growing literature examining related questions using electroencephalographic (EEG) and magnetoencephalographic (MEG) techniques. Much of this work has focused on the mu rhythm, which occurs in the alpha frequency range at central electrode sites overlying sensorimotor cortices. Mu rhythm suppression has been documented during the experience of touch (Cheyne et al., 2003; Gaetz and Cheyne, 2006), and during the observation of social touch (Peled-Avron et al., 2016) and pain (Cheng et al., 2008; Perry et al., 2010; Riečanský et al., 2015; Whitmarsh et al., 2011). Furthermore, viewing painful stimulation elicits stronger mu desynchronization than watching non-painful tactile stimulation (Höfle et al., 2013; Perry et al., 2010; Whitmarsh et al., 2011; Yang et al., 2009). 
These findings of neural activation during observation of touch have been used to support the broader notion that we relate to others' experiences in part through mapping the sensations (e.g., tactile sensations) experienced by other people onto our own sensory representations (Keysers et al., 2010). However, despite much interest in this idea, the necessary conditions under which such shared activations might occur remain poorly understood, and the specific role of activation of somatosensory cortex in understanding others' somatic states is not clear (Chan and Baker, 2015; Lamm et al., 2015). One limitation of prior work in this area is that most studies have examined changes in brain activity at a relatively coarse temporal resolution. Different stages of a "touching" event (either seen or felt), such as the anticipation of stimulation, the motion of an object or a hand approaching the stimulation location, and the contact itself, might be associated with the activation of different neural circuitries. To explore the specifics of shared somatosensory activations, it may be useful to isolate and examine these various stages. In this respect, the high level of temporal resolution provided by EEG methods can be helpful in unraveling the complexities of the similarities and differences in cortical activity between somatosensory stimulation to oneself and stimulation of others.

In the current study we take a novel approach by applying EEG techniques to examine the anticipatory processes that occur following a cue signaling the impending presentation of a tactile stimulus to self or other. Although prior studies of shared somatosensory activations have generally not considered anticipatory responses, there is a growing literature on the dynamics of EEG responses during anticipation of sensory stimulation (Arnal and Giraud, 2012). Another novel aspect of the current study comes 
from the fact that prior work in this area has often employed short video stimuli depicting another person being touched. Here we focus on anticipation of tactile stimulation using a protocol in which touch was not observed, but instead was signaled through a visual cue that indicated the impending delivery of a tactile stimulus to oneself or to another person. Very few studies have employed this kind of protocol. An fMRI study on empathy for pain (Singer et al., 2004) employed a visual cue to signal that an impending painful or non-painful electrical stimulus would be delivered a few seconds later to the hand of either oneself or one's partner. Responses to the painful stimulation being delivered to the partner were noted in the anterior cingulate cortex (ACC) and anterior insula (AI), areas associated with empathy, arousal, and anxiety (Lamm \& Singer, 2010), However, due to a lack of temporal resolution of fMRI data, the specific anticipatory aspects of these responses were not clear. It also remains an open question whether there are commonalities in neural responses over somatosensory areas during anticipation of tactile stimulation being delivered to oneself or another person. In the present study we examine this question by tracing changes in EEG alpha band power and changes in slow potentials in the event-related potential (ERP) during anticipation of an impending tactile stimulus.

There is increasing interest in the role of the alpha rhythm $(8-14 \mathrm{~Hz}$ in adults) in anticipatory neural processes (Arnal and Giraud, 2012; Anderson and Ding, 2011; Haegens et al., 2012; Jones et al., 2010; van Dijk et al., 2008). Much of the work in this area has involved the anticipation of visual targets in spatial attention tasks, in which a cue signals the impending appearance of the target and further indicates the location (e.g., the left vs. right side of the monitor screen) at which the target can be expected to 
appear. During the epoch between the cue and the target, an event-related desynchronization (ERD) in alpha power is typically seen over occipital scalp sites contralateral to the cued location (Kelly et al., 2006; Sauseng et al., 2005; Thut et al., 2006; Worden et al., 2000), with some studies also reporting an ipsilateral event-related synchronization (ERS) of the posterior alpha rhythm (Rins et al., 2009). With regard to anticipation of tactile stimuli, a similar desynchronization following a cue has been reported for the alpha-range mu rhythm over central electrode sites. For instance, if a cue signals impending stimulation of the left hand, an anticipatory desynchronization is seen in the alpha frequency range over the contralateral (right) central electrode site. The extent of anticipatory mu desynchronization is correlated with tactile discrimination and detection accuracy (Anderson and Ding, 2011; Jones et al., 2010; LinkenkaerHansen, 2004; Schubert et al., 2009), as well as with the expected intensity of tactile stimulation (Höfle et al., 2013). Across both visual and somatosensory modalities, the anticipatory desynchronization of alpha-range rhythms (over posterior and central sites respectively) has been proposed to indicate an attentional mechanism involving an increase in local sensory cortex excitability, which increases the perceptual salience of incoming tactile stimuli (Foxe and Snyder, 2011).

In addition to the alpha rhythm, another anticipatory component in the EEG signal is a slow negative potential that is commonly known as the contingent negative variation (CNV; Brunia et al., 2012; Tecce, 1972; Van Boxtel and Bocker, 2004; Walter, 1964). The CNV is a slow buildup of a negative-going EEG potential during the interval between a warning/cue stimulus (S1) and a target stimulus (S2), which has been interpreted as reflecting the deployment of attention (Babiloni et al., 2003; Bickel et al., 
2012; Hamano et al., 1997; Tecce, 1972), timing processes (e.g. gauging expected duration of cue-target interval; Jang et al., 2016; Macar and Vidal, 2009; Mento, 2013; van Rijn et al., 2011) or changes in alertness (Pauletti et al., 2014; Posner, 2008). The CNV is commonly observed over frontal-central regions prior to the delivery of auditory or tactile stimuli (Chennu et al., 2013; Kononowicz and Penney, 2016; Pauletti et al., 2014), and has a strong parietal-occipital distribution preceding visual targets (Gomez, et al., 2007). The amplitude of the CNV is modulated by several factors, including whether the target stimulus (S2) requires a motor response, which elicits larger CNV amplitudes than non-motor responses (Bareš et al., 2007). Other studies have reported that the complexity of tasks is associated with CNV amplitude (Kranczioch et al., 2010; Cui et al., 2000). CNV amplitude is also reduced by insertion of distractors (Tecce \& Scheff, 1969), but not attenuated by stimuli repetitions (Pauletti et al., 2014).

Studying mu ERD and the CNV has yielded useful insights into the neural processes that are active during the anticipation and subsequent perception of somatosensory stimulation. However, whether similar electrophysiological responses can also be observed during the anticipation of other peoples' somatosensory experiences has not yet been investigated. Vicarious activations elicited in somatosensory cortex during direct observation of tactile stimulations to others suggested an important role of somatosensory cortex in social perception and empathy (Keysers \& Gozzola, 2009; Schaefer et al., 2009). However, the necessary conditions for such vicarious activation, as well as the time course of such activation, remain unknown. It is possible that social perception also influences anticipatory neural activities prior to the onset of actual tactile events, which would suggest a role for 
attention networks and thalamo-cortical circuitries. In the present study, EEG was recorded from undergraduate participants while they completed a simple tactilediscrimination task with an experimenter. Prior to each tactile stimulus, cues were presented on a screen signaling whether tactile stimulation would be presented to the participant, their partner, or neither. Analyses focused on changes in the mu rhythm and the amplitude of the anticipatory CNV between the onset of the visual cue and the tactile target stimulus. If vicarious activation in response to others' somatosensory states occurs during the anticipation of a tactile stimulus delivered to another person, mu rhythm ERD and a CNV potential would not only be observed when participants are expecting tactile stimulation to themselves, but would also be measureable during anticipation of stimulation of their task partner.

The insights gleaned from this study can add to our understanding of social influences on anticipatory neural processes, and can expand current understanding on vicarious activation and social empathy. A supplementary goal of the current analyses was also to examine the relations between mu rhythm ERD and the anticipatory negativities. Despite the fact that these two anticipatory measures are usually elicited in similar experimental paradigms and occur over similar time frames, the relation between them is not clear (Green and McDonald, 2010; Grent-'T-Jong et al., 2011).

\section{Results}

2.1 Behavioral results 
Throughout the task, participants received "short" ( 3 successive) or "long" (5 successive) tactile pulses. For each trial which they received tactile stimulation, they were asked to report whether the stimulus was of the long or short variety. Mean behavioral accuracy for discriminating the tactile target stimuli (long vs. short pulses) was $93.71 \%(S D=3.71)$, indicating that participants were engaged with the task.

\subsection{EEG band power results}

In order to examine event-related changes in alpha band power, event-related spectral perturbation (ERSP) in the $8-14 \mathrm{~Hz}$ frequency band was compared using ANOVA across three conditions (self/other/nobody) for the $-1000 \mathrm{~ms}$ to $-500 \mathrm{~ms}$ window relative to delivery of the tactile stimulus. As shown in Figure 1, for anticipation of stimulation to the right hand, significant differences in alpha ERSP between conditions were apparent at various electrodes across frontal, central, and parietal recording sites (specifically electrodes Fz, FC1, C3, Cz, C4, CP5, CP1, CP2, P3, and P7; $p<.01$ ). Prior to stimulation of the left hand, significant differences in alpha ERSP between conditions were observed at electrodes C3, C4, CP5, and CP6 $(p<0.05)$. As seen on the topographic maps in Figure 1, these significant differences were driven by a greater desynchronization when participants were anticipating tactile stimulation to themselves ("self") compared to when they were anticipating tactile stimulation to their task partner ("other") or to no one ("nobody"). Pair-wise comparisons showed no significant differences in alpha ERSP fluctuation between the "other" and "nobody" conditions at any of the electrodes prior to left and right hand stimulation. 
$<$ insert figure 1 around here>

Further analysis of ERSP in the alpha band focused on electrodes C3 and C4, in order to ascertain the dynamics of the sensorimotor mu rhythm during anticipation of tactile stimulation. Figure 2 shows mean $\mathrm{mu}(8-14 \mathrm{~Hz})$ power at $\mathrm{C} 3$ and $\mathrm{C} 4$ from the onset of the cue to $500 \mathrm{~ms}$ post-stimulus. Analyses focused on the time window between $-1000 \mathrm{~ms}$ and $-500 \mathrm{~ms}$ prior to tactile stimulation. Mean mu rhythm power in this time window was computed and was used as the dependent variable in a repeated-measures ANOVA with the following factors: Condition (self/other/nobody), Electrode (C3/C4), and Block Type (left hand/right hand). The mean ERSP values from this analysis are shown in Figure 3.

Results revealed a significant main effect of Condition $(F(2,44)=79.808, p<$ 0.001), and a significant interaction between Block Type $\times$ Electrode $(F(1,22)=8.081, p$ $=0.009)$. In terms of the main effect, Tukey's post-hoc tests showed that mu desynchronization for the "self" condition was significantly larger than for the "nobody" and "other" conditions $(p<0.001)$, with no difference between the "nobody" and "other" conditions $(p=0.982)$. Concerning the interaction effect, follow-up repeated-measures ANOVAs for each Block Type showed significant main effects of Electrode only for blocks in which stimuli were presented to the right hand $(F(1,22)=8.499, p=0.008)$, during which mu desynchronization was greater at C3 than at C4. No significant difference in mu desynchronization was found between $\mathrm{C} 3$ and $\mathrm{C} 4$ during blocks when the tactile stimuli were delivered to the left hand $(F(1,22)=0.636, p=0.434)$. 
< insert figure 2 around here
$<$ insert figure 3 around here

\subsection{ERP results}

Figure 4 shows the topographic distribution of the anticipatory negativity (CNV) in the ERP that was elicited following the cue. For blocks in which stimuli were delivered to the right hand, the magnitude of the CNV was significantly greater for the "self" condition than for the "nobody" or "other" conditions at specific frontal, central, and central-parietal recording sites (electrodes $\mathrm{FC} 1, \mathrm{C} 3, \mathrm{Cz}, \mathrm{CP} 5, \mathrm{CP} 1$, and $\mathrm{CP} 2 ; \mathrm{p}<0.001$ ). During blocks in which stimuli were delivered to the left hand, a significantly stronger CNV was observed over central sites (C3 and C4) for the "self" condition compared with the "nobody" and "other" conditions $(p<0.01)$. Figure 5 shows the ERPs that were elicited at C3 and C4 during the interval between the onset of the visual cue and the onset of the subsequent tactile stimulus.

Mean ERP amplitude was computed across a $800 \mathrm{~ms}$ window from $-900 \mathrm{~ms}$ to $100 \mathrm{~ms}$ for each subject, and then used as the dependent variable in a repeatedmeasures ANOVA with the following factors: Condition (self/other/nobody), Electrode (C3/C4), and Block Type (left hand/right hand). The mean amplitude values from this analysis are shown in Figure 6.

Results showed a significant main effect of Condition $(F(2,44)=25.486, p<$ 0.001), and a significant 3-way interaction between Condition $\times$ Block Type $\times$ Electrode $(F(2,44)=10.734, p<0.001)$. Tukey's post-hoc tests showed that the anticipatory negativity for the "self" condition was significantly larger than for both the "other" and 
"nobody" conditions $(p<0.001)$, while no difference was found between the "other" and "nobody" conditions $(p=0.555)$. To follow up the significant interaction between Condition, Block Type, and Electrode, two-way analyses of variance were conducted for each condition. A significant interaction between Block Type $\times$ Electrode was found for the "self" condition $(F(1,22)=19.457, p<0.001)$, but there were no significant findings for the "other" and "nobody" conditions $(p>0.05)$. Pairwise comparisons with false discovery rate correction revealed that when the tactile stimuli were delivered to the participant's right hand, CNV amplitude was significantly larger at C3 than C4 ( $p=$ 0.012). There was no significant difference in amplitude between C3 and C4 during blocks when stimuli were delivered to the participant's left hand $(p=0.962)$.

$<$ insert figure 4 around here $>$

$<$ insert figure 5 around here $>$

$<$ insert figure 6 around here>

A final set of analyses was conducted to explore the correlations between the extent of alpha ERD and the amplitude of the anticipatory ERP, again with a focus on central electrodes. The mean ERP amplitudes and mean ERSP between $-1000 \mathrm{~ms}$ to Oms (tactile stimulus onset time) for the "self" condition were extracted and subjected to Pearson's $r$ correlation statistics. For blocks in which the tactile stimuli were presented to the right hand, correlations between these two measures approached statistical significance $(r=-0.381, p=0.073$ at C3 and $r=-0.360, p=0.091$ at C4). For stimulation of the left hand, correlations were more clearly nonsignificant $(r=0.047, p=$ 0.830 at $\mathrm{C} 3$ and $r=-0.119, p=0.589$ at $C 4)$. 


\section{Discussion}

There is sustained interest in the involvement of somatosensory processes in social perception (Keysers et al. 2010). This line of research has often involved testing whether the neural systems involved in somatosensory processing are activated in response to tactile stimulation of another person (Blakemore et al., 2005; Ebisch et al., 2008; Jackson et al., 2006; Keysers et al., 2004; Kuehn et al., 2014; Schaefer et al., 2009). Much of this prior research on shared activation of somatosensory circuits has focused on recording brain activity during the observation of touch to others. The current study relied less on visual aspects, instead using a cued tactile stimulation paradigm to explore EEG activity elicited during the anticipation of a tactile stimulus that was not observed. More specifically, a visual cue signaled the delivery of an impending tactile stimulus, and anticipatory EEG activity across the cue-target interval was compared across three different conditions: During anticipation of tactile stimulation of the participant's own hand, during anticipation of tactile stimulation delivered to another person's hand, or during anticipation of a stimulus that did not result in tactile stimulation to either person.

Analyses of the EEG data focused on two particular anticipatory responses event-related desynchronization in the alpha frequency band and the contingent negative variation in the event-related potential. Alpha band ERD as well as a CNV response were clearly observable while participants anticipated the delivery of tactile stimulation to their own hands, which is consistent with results from previous EEG studies on anticipation of somatosensory stimuli (Babiloni et al., 2003; Foxe and Snyder, 
2011). Similar ERD and CNV responses were not observed in the other two conditions. In the statistical comparisons between conditions, it was apparent that the differences in alpha-range desynchronization and CNV amplitude between the "self" condition and the other two conditions were localized over central and central parietal recording sites. Follow-up analyses focused on the central electrodes $\mathrm{C} 3$ and $\mathrm{C} 4$, which roughly overly the sensorimotor hand areas and at which the alpha-range mu rhythm is typically recorded.

For mu ERD and CNV amplitude at central sites, hemispheric differences in the anticipatory responses were dependent on whether stimuli in a given block were presented to the left or right hand. During blocks in which stimuli were delivered to the right hand, CNV amplitude and mu ERD were stronger at the contralateral central site than at the ipsilateral site. There was no significant hemispheric difference in CNV and mu ERD during blocks in which stimuli were delivered to the left hand. We suspect that this lack of lateralization effects during the left hand blocks may be related to the fact that the dominant hand all the participants in this study was the right hand. Furthermore, the pre-target cues in the study were not informative about which hand would receive tactile stimulation. Instead, participants received that information at the beginning of each block. Therefore, the spatial location of tactile stimuli stimulation may not be as salient as in other EEG studies in which contralateral activity was reported. These studies typically presented spatial location cues on a trial-by-trial basis, with the participant directing attention to either their left or right hand prior to each tactile stimulus (Anderson and Ding, 2011; Eimer, et al., 2003; Haegens, et al., 2012). 
Our goal in this study of exploring possible social influences on brain activity during anticipation of tactile stimuli was inspired by two observations. One was the vicarious activation in somatosensory cortices when participants witness stimulation to other people or actions of others, in terms of BOLD signals in fMRI studies and mu ERD in EEG studies (Keysers, et al., 2010; Peled-Avron et al., 2016). The other observation was the anticipatory brain potentials that have been documented to occur prior to the delivery of tactile stimulation to one's own hand (Eimer et al., 2003; Linkenkaer-Hansen, 2004; Schubert et al., 2009). In the present study we asked whether participants might also exhibit signs of these anticipatory potentials if stimulation was delivered to another person's hand. However, the results clearly showed that an anticipatory mu ERD as well as the CNV response only occurred for the "self" condition, and not for the "other" condition, which in turn did not differ from a condition in which nobody received tactile stimulation.

In terms of reasons why anticipatory EEG and ERP responses were not observed for the "other" condition, we raise two possibilities. Firstly, studies that reported vicarious activation in primary or secondary somatosensory cortices (SI/SII) often involved participants viewing others' hands or feet being touched (Blakemore et al., 2005; Ebisch et al., 2008; Jackson et al., 2006; Keysers et al., 2004; Kuehn et al., 2014; Schaefer et al., 2009). Therefore, directly witnessing the somatosensory events or depictions of stimulation to body parts may be a necessary component in evoking vicarious anticipatory activation in somatosensory cortices (Keysers et al., 2010, for review). The absence of a concurrent visual depiction of the tactile stimulation in the 
current study may be one of the reasons why we did not observe activations during the anticipation of stimulation to another person.

A second possibility for the lack of anticipatory components seen prior to stimulation of the other person is the lack of an interpersonal connection between the participant and their task partner. In the current study, the participants and their partner (who was an experimenter) had the same instructions to identify the type of tactile stimulus (short vs. long) that they received on a given trial. The task instructions had emphasized that that participant and their partner were working together to achieve the best possible joint score on the tactile discrimination task. However, this instruction alone may not have been effective at promoting a particular connection between the participant and the other person. Participants were not aware of the accuracy or inaccuracy of their partner's responses, and the discrimination task was relatively easy. Therefore, this setup might not be as effective as other designs, such as the joint Simon task (Sebanz et al., 2003) in promoting a sense of working together towards a shared goal. Future studies on anticipatory activations to stimulation of self vs. other could use variations on established joint tasks to promote a sense of connection between the participants. Another possibility is to employ participants who share strong social bonds with each other, such as friends or couples. This suggestion also relates to findings concerning empathic responses to tactile stimulation of others (Lamm et al., 2015; Singer et al., 2004), which can also involve responses in brain networks related to affective processes.

Correlational analyses explored the relations between the extent of the anticipatory mu desynchronization and the amplitude of the CNV response that both occurred during the window between the cue and the tactile stimulus. Although some 
correlations were close to statistical significance, the overall relations were relatively weak, suggesting that individual differences in anticipatory mu desynchronization do not clearly map onto individual differences in CNV amplitude. This null finding is consistent with results from earlier studies examining anticipatory brain potentials and alpha desynchronization prior to the presentation of a visual target (Green and McDonald, 2010; Grant T'Jong et al., 2011). In the current study, there were further differences between mu desynchronization and the CNV in terms of their temporal and topographic distributions. For instance, visual inspection of the waveforms suggests that the onset of the CNV was around $200 \mathrm{~ms}$ later than the onset of mu desynchronization. In addition, the CNV had a more frontal distribution than the mu response, which was observed mainly at central-parietal recording sites.

One issue for further investigation concerns similarities and differences in the lateralization of the mu desynchronization and CNV responses. Both types of responses were most clearly lateralized to the contralateral central electrode during blocks of trials in which stimuli were presented to the right hand. For mu ERD, this contralateral effect was found regardless of condition (self/other/nobody) while for the CNV, the lateralization was only apparent during the "self" condition in which stimuli were delivered to the participant's right hand. These differences suggest that anticipatory lateralization of the mu rhythm may be less dependent on whether the anticipated target stimulus is delivered to self or other (or nobody), while the CNV response is more sensitive to anticipation of sensory effects that are specifically directed toward the self.

Although mu ERD and the CNV can be elicited in similar paradigms, and both components likely involve attentional mechanisms, they may reflect different functional 
roles, with possible non-linear interactions. Current consensus suggests that desynchronization of alpha-range oscillations reflect an attention-modulated inhibition/gating mechanism, which stems from rhythmic GABAergic activity generated by local sensory cortex and thalamo-cortical circuitries (de Munck et al., 2009; Foxe and Snyder, 2011; Klimesch, 2012; Klimesch et al., 2007; Ritter et al., 2009; Sadaghiani \& Kleinschmidt, 2016). In terms of the origin of the mu rhythm specifically, various studies using high-density EEG or MEG have identified somatosensory cortex as one source of mu ERD (Anderson and Ding, 2011; Cheyne et al., 2003; Gaetz and Cheyne, 2006; Whitmarsh and Jensen, 2011). In comparison, the functional role and neural generators of CNV are less well understood, partly because the CNV is often intermingled with readiness potentials that reflect motor preparation (Cui et al., 2000), and with other stimulus-preceding negativities related to feedback and reward (Kotani et al., 2015).

Several studies have suggested that the CNV is generated by a combination of control mechanisms involving a frontal-parietal attentional network as well as local sensory cortices (Hammano, et al., 1997; Giesbrecht et al., 2006; Gómez et al., 2007; Macaluso et al., 2003; Nagai, et al., 2004; Pauletti et al., 2014). However, while their functional and anatomical mechanisms remain unclear, there may be some complex non-linear interactions between alpha rhythms and slow potentials such as the CNV (Kononowicz and Penney, 2016; Mazaheri and Jensen, 2008; Van Boxtel and Bocker, 2004). Further studies using high-density EEG or simultaneous EEG and fMRI recording might help disentangle the relations between anticipatory CNV and mu ERD and may shed further light on the complexities of anticipatory brain responses. A few fMRI studies on perceptual anticipatory activities have reported activations in multiple brain areas 
during anticipation of sensory stimuli, including modality-specific contralateral primary sensory cortices, as well as right anterior insular and frontal-parietal attention networks (Carlsson et al., 2000; Langner et al., 2011).

In conclusion, this study tested whether anticipatory responses to impending tactile stimulation could be detected before tactile stimulation of oneself or of another person. The results revealed robust anticipatory mu desynchronization and CNV responses before participants received tactile stimulations to their own hand. We did not observe clear anticipatory signals prior to tactile stimulation being delivered to another person's hand, possibly due to a lack of visual depictions of somatosensory events and the limited interpersonal connections between participants and their task partners. Finally, the absence of a clear correlation between CNV and mu ERD magnitudes, as well as subtle differences in temporal and topographic distributions between CNV and mu ERD, suggested different, but possibly overlapping, neural mechanisms underlying these two signatures of anticipatory processing.

\section{Experimental procedures}

\subsection{Participants}

A total of 25 undergraduate participants (18 females, mean age $=20.57, \mathrm{SD}=$ 2.54) participated in the study. All were right-handed, with normal or corrected-to-normal vision, and had no history of neurological abnormality. Data from 23 participants were used for final analysis. One dataset was excluded because of hardware problems, and another was excluded due to insufficient artifact-free trials (less than 25 trials/condition). 


\subsection{Tactile stimulation}

Tactile stimuli were delivered to the distal tip of participants' left and right index fingers using an inflatable membrane mounted in a plastic casing. This stimulation method has been used in prior EEG and MEG studies (Pihko et al., 2009; Saby et al., 2015). The membrane was inflated by a short burst of compressed air delivered via flexible polyurethane tubing ( $3 \mathrm{~m}$ length, $3.2 \mathrm{~mm}$ outer diameter). The compressed air delivery was controlled by STIM stimulus presentation software in combination with a pneumatic stimulator unit (both from James Long Company) and an adjustable regulator that restricted the airflow to 100psi. To generate each tactile stimulus, the STIM software triggered a solenoid in the pneumatic stimulator to open for $10 \mathrm{~ms}$. Expansion of the membrane started $20 \mathrm{~ms}$ after the trigger onset, and peaked $20 \mathrm{~ms}$ later. The task used was a tactile discrimination task in which $50 \%$ of the stimuli were "short" (3 tactile pulses in rapid succession) and $50 \%$ of the stimuli were "long" (a series of 5 pulses).

\subsection{Procedure}

Participants were fitted with an EEG cap, and were seated comfortably in front of a computer next to an experimenter, who acted as their partner for the tactile discrimination task. The experimenter was also fitted with tactile stimulators on their left and right index fingers and the participant was given instructions about the task. At the start of each trial, participants viewed a fixation image that lasted for $2000 \mathrm{~ms}$, followed by a visual cue that signaled the imminent delivery of a tactile stimulus (see Figure 1). Three different kinds of cues were presented: 1) a circle, which indicated that the tactile stimulus would delivered to the participant; 2) a square, which indicated that the stimulus would be delivered to the experimenter (other); 3) a triangle, which indicated 
that neither the participant or the experimenter would receive any stimulus (see Figure 1). For trials in which neither person was stimulated, participants were told that similar pulses would be sent through a polyurethane tube that ran between the participant and experimenter and was not attached to anything.

The delay between the onset of the visual cue and the delivery of the tactile stimulation was $1500 \mathrm{~ms}$. During this interval, white noise was played through open field speakers in order to mask any sounds produced by the solenoids. At 500 ms following the onset of the tactile stimulation, a screen showing "Respond" was displayed. For trials in which they received tactile stimulation, participants needed to verbally report whether they received a short pulse or a long pulse. The verbal reports were recorded for later analysis. The experimenter also provided similar verbal reports for the relevant trials in which he or she was cued to receive tactile stimulation. These verbal reports occurred within a 2000 ms window following the display of the "Respond" screen. There was then a 1000 ms delay during which the screen was black, following which the fixation point appeared to signal the beginning of a new trial.

Stimulation of the left hand and the right hand was carried out in separate blocks. Each block consisted of 54 trials (18 trials for each of the self/other/nobody conditions in random order) and there were six blocks in total ( 3 for the left hand and 3 for the right hand). Prior to the beginning of the experiment, participants were informed that they would be carrying out the task together with the other person, and that it was the joint responsibility of both partners to achieve a high accuracy rate on the task.

$<$ insert figure 7 around here> 


\subsection{EEG recording}

EEG signals were recorded from 29 electrode sites (Fp1, Fp2, F3, F4, Fz, F7, F8, C3, C4, Cz, T7, T8, P3, P4, Pz, P7, P8, O1, O2, M1, M2, Fc1, Fc2, Fc5, Fc6, Cp1, Cp2, Cp5, Cp6) using a Lycra stretch cap (Electro-Cap, Eaton, OH, USA) placed according to the international $10-20$ system. Vertical electro-oculogram (EOG) activity was collected from electrodes placed above and below the left eye. Scalp electrode impedances were kept under $25 \mathrm{~K} \Omega$. All EEG and EOG channels were continuously recorded with a sampling frequency of $512 \mathrm{~Hz}$. Hardware bandpass filter settings were $0.1 \mathrm{~Hz}$ (high-pass) and $100 \mathrm{~Hz}$ (low-pass) and the gain was 4000 for EEG channels and 1000 for EOG. The EEG signals were collected referenced to Cz with an AFz ground, and were re-referenced offline to the average of the left and right mastoids.

\subsection{Data analysis}

\subsubsection{Pre-processing of EEG data}

EEG analysis was performed using the EEGLAB 13.5.4b toolbox (Delorme and Makeig, 2004) implemented in MATLAB. Following re-referencing to the average of the left and right mastoids, epochs were extracted from the continuous EEG data. Each epoch was 3000 ms in duration, beginning 500ms before cue onset and ending 1000ms after the onset of the tactile stimulus. Independent component analysis (ICA) was conducted to remove eye movement artifacts (Hoffmann \& Falkenstein, 2008). Visual inspection of the EEG signal was used to reject epochs containing movement artifact. The mean number of artifact-free trials per condition was $48(S D=4.72)$, There was no significant difference in the number of usable trials across conditions $(p=0.160)$. 


\subsubsection{Time-frequency Analysis}

Time-frequency decompositions of single trial data were conducted using eventrelated spectral perturbation (ERSP) analysis (Makeig, 1993), for a 2500 ms window that ran from $-2000 \mathrm{~ms}$ prior to the onset of the tactile stimulus to $500 \mathrm{~ms}$ after tactile stimulation onset. ERSP was computed using a Morlet wavelet decomposition over a frequency range of 5 to $25 \mathrm{~Hz}$, with 100 overlapping windows starting with a 3-cycle wavelet at the lowest frequency. The baseline was defined as the $500 \mathrm{~ms}$ period immediately before cue onset (i.e., -1500 to $-2000 \mathrm{~ms}$ prior to the onset of tactile stimulation). Event-related desynchronization (ERD) is indicated as an ERSP decrease relative to the baseline. In order to assess anticipatory effects induced by the different cues, changes in alpha $(8-14 \mathrm{~Hz})$ ERSP were compared between the three conditions (self/other/nobody) across all electrodes using two different levels of significance $(p<$ 0.05 and $p<0.01)$. Results were corrected for multiple comparisons by controlling the false discovery rate (FDR). To examine lateralization effects during anticipation, repeated measures ANOVA with Greenhouse-Geisser correction was used to compare averaged alpha ERSP during a window of $-1000 \mathrm{~ms}$ to $-500 \mathrm{~ms}$ at the left and right central electrodes (C3 \& C4).

\subsubsection{ERP analysis}

To prepare the data for ERP analysis, the artifact-free epochs were filtered with a low-pass filter at $30 \mathrm{~Hz}$, and were then averaged and baseline corrected relative to the $500 \mathrm{~ms}$ pre-cue baseline. The contingent negative variation (CNV) was identified as the slow negative wave between the cue and tactile stimuli. The calculation of mean amplitude was calculated by averaging across a $800 \mathrm{~ms}$ window from $-900 \mathrm{~ms}$ to $-100 \mathrm{~ms}$ 
prior to the unset of tactile stimuli for electrodes C3 and C4. Repeated-measures ANOVAs on mean CNV amplitudes were conducted with the factors Electrode (C3/C4), Hand (left/right), and Condition (self/other/nobody). Greenhouse-Geisser adjustments to the degrees of freedom were performed. Post-hoc analysis was conducted using pairwise t-test with FDR corrections.

\section{Acknowledgements}

The authors thank Zoe Kearns and Edna Ollquist for their help with data collection. The writing of this article was supported in part by awards from NIH (1R21HD083756) and NSF (BCS-1460889).

\section{REFERENCES}

Anderson, K.L., Ding, M., 2011. Attentional modulation of the somatosensory mu rhythm. Neuroscience 180, 165-180.

Arnal, L. H., \& Giraud, A. L. 2012. Cortical oscillations and sensory predictions. Trends in Cognitive Sciences, 16, 390-398.

Babiloni, C., Brancucci, A., Babiloni, F., Capotosto, P., Carducci, F., Cincotti, F., ArendtNielsen, L., Chen, A.C.N., Rossini, P.M., 2003. Anticipatory cortical responses during the expectancy of a predictable painful stimulation. A high-resolution electroencephalography study. Eur. J. Neurosci. 18, 1692-1700.

Bareš, M., Nestrašil, I., Rektor, I., 2007. The effect of response type (motor output versus mental counting) on the intracerebral distribution of the slow cortical 
potentials in an externally cued (CNV) paradigm. Brain Res. Bull. 71, 428-435.

Bickel, S., Dias, E.C., Epstein, M.L., Javitt, D.C., 2012. Expectancy-related modulations of neural oscillations in continuous performance tasks. Neuroimage $62,1867-1876$.

Blakemore, S.J., Bristow, D., Bird, G., Frith, C., Ward, J., 2005. Somatosensory activations during the observation of touch and a case of vision-touch synaesthesia. Brain 128, 1571-1583.

Brunia, C. H., van Boxtel, G. M., \& Böcker, K. B., 2012. Negative slow waves as indices of anticipation: The bereitschaftspotential, the contingent negative variation, and the stimulus-preceding negativity. The Oxford handbook of event-related potential components, 189-207.

Cappe, C., Thut, G., Romei, V., Murray, M.M., 2010. Auditory-Visual Multisensory Interactions in Humans: Timing, Topography, Directionality, and Sources. J Neurosci 30, 12572-12580.

Carlsson, K., Petrovic, P., Skare, S., Petersson, K.M., Ingvar, M., 2000. Tickling expectations: neural processing in anticipation of a sensory stimulus. J. Cogn. Neurosci. 12, 691-703

Chan, A.W.-Y., Baker, C.I., 2015. Seeing Is Not Feeling: Posterior Parietal But Not Somatosensory Cortex Engagement During Touch Observation. J. Neurosci. 35, $1468-1480$.

Cheng, Y., Yang, C.Y., Lin, C.P., Lee, P.L., Decety, J., 2008. The perception of pain in others suppresses somatosensory oscillations: A magnetoencephalography study. Neuroimage 40, 1833-1840. 
Chennu, S., Noreika, V., Gueorguiev, D., Blenkmann, A., Kochen, S., Ibáñez, A., Owen, A.M., Bekinschtein, T. a, 2013. Expectation and attention in hierarchical auditory prediction. J. Neurosci. 33, 11194-205.

Cheyne, D., Gaetz, W., Garnero, L., Lachaux, J.P., Ducorps, A., Schwartz, D., Varela, F.J., 2003. Neuromagnetic imaging of cortical oscillations accompanying tactile stimulation. Cogn. Brain Res. 17, 599-611.

Costantini, M., Galati, G., Romani, G.L., Aglioti, S.M., 2008. Empathic neural reactivity to noxious stimuli delivered to body parts and non-corporeal objects. Eur. J. Neurosci. 28, 1222-1230.

Cui, R.., Egkher, A., Huter, D., Lang, W., Lindinger, G., Deecke, L., 2000. High resolution spatiotemporal analysis of the contingent negative variation in simple or complex motor tasks and a non-motor task. Clin. Neurophysiol. 111, 1847-1859.

de Munck, J.C., Gonçalves, S.I., Mammoliti, R., Heethaar, R.M., Lopes da Silva, F.H., 2009. Interactions between different EEG frequency bands and their effect on alpha-fMRI correlations. Neuroimage 47, 69-76.

Delorme, A., Makeig, S., 2004. EEGLAB: An open source toolbox for analysis of singletrial EEG dynamics including independent component analysis. J. Neurosci. Methods 134, 9-21.

Dolk, T., Hommel, B., Colzato, L.S., Schütz-Bosbach, S., Prinz, W., Liepelt, R., 2014. The joint Simon effect: a review and theoretical integration. Front. Psychol. 5, 974.

Ebisch, S.J.H., Perrucci, M.G., Ferretti, A., Del Gratta, C., Romani, G.L., Gallese, V., 2008. The sense of touch: Embodied simulation in a visuotactile mirroring 
mechanism for observed animate or inanimate touch. J. Cogn. Neurosci. 20, 16111623.

Foxe, J.J., Snyder, A.C., 2011. The role of alpha-band brain oscillations as a sensory suppression mechanism during selective attention. Front. Psychol. 2, 1-13.

Gaetz, W., Cheyne, D., 2006. Localization of sensorimotor cortical rhythms induced by tactile stimulation using spatially filtered MEG. Neuroimage 30, 899-908.

Giesbrecht, B., Weissman, D.H., Woldorff, M.G., Mangun, G.R., 2006. Pre-target activity in visual cortex predicts behavioral performance on spatial and feature attention tasks. Brain Res. 1080, 63-72.

Gómez, C.M., Flores, A., Ledesma, A., 2007. Fronto-parietal networks activation during the contingent negative variation period. Brain Res. Bull. 73, 40-47.

Grent-'T-Jong, T., Boehler, C.N., Kenemans, J.L., Woldorff, M.G., 2011. Differential functional roles of slow-wave and oscillatory-alpha activity in visual sensory cortex during anticipatory visual-spatial attention. Cereb. Cortex 21, 2204-2216.

Haegens, S., Luther, L., Jensen, O., 2012. Somatosensory Anticipatory Alpha Activity Increases to Suppress Distracting Input. J. Cogn. Neurosci. 24(3), 677-685.

Hamano, T., Lüders, H.O., Ikeda, A., Collura, T.F., Comair, Y.G., Shibasaki, H., 1997. The cortical generators of the contingent negative variation in humans: A study with subdural electrodes. Electroencephalogr. Clin. Neurophysiol. - Evoked Potentials 104, 257-268.

Hoffmann, S., Falkenstein, M., 2008. The correction of eye blink artefacts in the EEG: A 
comparison of two prominent methods. PLoS One 3 (8), e3004.

Höfle, M., Pomper, U., Hauck, M., Engel, A.K., Senkowski, D., 2013. Spectral signatures of viewing a needle approaching one's body when anticipating pain. Eur. J. Neurosci. 38, 3089-3098.

Jackson, P.L., Brunet, E., Meltzoff, A.N., Decety, J., 2006. Empathy examined through the neural mechanisms involved in imagining how I feel versus how you feel pain. Neuropsychologia 44, 752-761.

Jang, J., Jones, M., Milne, E., Wilson, D., Lee, K.H., 2016. Contingent negative variation (CNV) associated with sensorimotor timing error correction. Neuroimage 127, 5866.

Jones, S.R., Kerr, C.E., Wan, Q., Pritchett, D.L., Ha, M., Moore, C.I., 2010. Modulation of the Mu Rhythm in Primary Somatosensory Cortex. J. Neurosci. 30, 13760 13765.

Kelly, S.P., Lalor, E.C., Reilly, R.B., Foxe, J.J., 2006. Increases in alpha oscillatory power reflect an active retinotopic mechanism for distracter suppression during sustained visuospatial attention. J. Neurophysiol. 95, 3844-51.

Keysers, C., Gazzola, V., 2009. Expanding the mirror: vicarious activity for actions, emotions, and sensations. Curr. Opin. Neurobiol. 19, 666-671.

Keysers, C., Kaas, J.H., Gazzola, V., 2010. Somatosensation in social perception. Nat. Rev. Neurosci. 11, 417-428.

Keysers, C., Wicker, B., Gazzola, V., Anton, J.L., Fogassi, L., Gallese, V., 2004. A 
touching sight: SII/PV activation during the observation and experience of touch. Neuron 42, 335-346.

Klimesch, W., 2012. Alpha-band oscillations, attention, and controlled access to stored information. Trends Cogn. Sci. 16, 606-617.

Klimesch, W., Sauseng, P., Hanslmayr, S., 2007. EEG alpha oscillations: The inhibition-timing hypothesis. Brain Res. Rev. 53, 63-88.

Kononowicz, T.W., Penney, T.B., 2016. The contingent negative variation (CNV): Timing isn’t everything. Curr. Opin. Behav. Sci. 8, 231-237. 2

Kotani, Y., Ohgami, Y., Ishiwata, T., Arai, J., Kiryu, S., Inoue, Y., 2015. Source analysis of stimulus-preceding negativity constrained by functional magnetic resonance imaging. Biol. Psychol. 111, 53-64.

Kranczioch, C., Mathews, S., Dean, P., Sterr, A., 2010. Task complexity differentially affects executed and imagined movement preparation: Evidence from movementrelated potentials. PLoS One 5.

Kuehn, E., Mueller, K., Turner, R., Sch??tz-Bosbach, S., 2014. The functional architecture of S1 during touch observation described with 7 T fMRI. Brain Struct. Funct. 219, 119-140.

Lamm, C., Meltzoff, A.N., Decety, J., 2009. How do we empathize with someone who is not like us? A functional magnetic resonance imaging study. J. Cogn. Neurosci. 22, $362-76$.

Lamm, C., Silani, G., Singer, T., 2015. Distinct neural networks underlying empathy for 
pleasant and unpleasant touch. Cortex 70, 79-89.

Lamm, C., Singer, T., 2010. The role of anterior insular cortex in social emotions. Brain Struct. Funct. 1-13. doi:10.1007/s00429-010-0251-3

Langner, R., Kellermann, T., Boers, F., Sturm, W., Willmes, K., Eickhoff, S.B., 2011. Modality-specific perceptual expectations selectively modulate baseline activity in auditory, somatosensory, and visual cortices. Cereb. Cortex 21, 2850-2862.

Linkenkaer-Hansen, K., 2004. Prestimulus Oscillations Enhance Psychophysical Performance in Humans. J. Neurosci. 24, 10186-10190.

Macaluso, E., Eimer, M., Frith, C.D., Driver, J., 2003. Preparatory states in crossmodal spatial attention: spatial specificity and possible control mechanisms. Exp. brain Res. 149, 62-74.

Macar, F., Vidal, F., 2009. Timing processes: an outline of behavioural and neural indices not systematically considered in timing models. Can. J. Exp. Psychol. 63, 227-239.

Makeig, S., 1993. Effects of Exposure to Pure Tones on Event-Related Dynamics of the EEG Spectrum. Electroencephalogr. Clin. Neurophysiol. 86, 283-293.

Mazaheri, A., Jensen, O., 2008. Asymmetric amplitude modulations of brain oscillations generate slow evoked responses. J. Neurosci. 28, 7781-7.

Mento, G., 2013. The passive CNV: carving out the contribution of task-related processes to expectancy. Front. Hum. Neurosci. 7, 827.

Nagai, Y., Critchley, H.D., Featherstone, E., Fenwick, P.B.C., Trimble, M.R., Dolan, 
R.J., 2004. Brain activity relating to the contingent negative variation: An fMRI investigation. Neuroimage 21, 1232-1241.

Pauletti, C., Mannarelli, D., Grippo, A., Curr??, A., Locuratolo, N., De Lucia, M.C., Fattapposta, F., 2014. Phasic alertness in a cued double-choice reaction time task: A Contingent Negative Variation (CNV) study. Neurosci. Lett. 581, 7-13.

Peled-Avron, L., Levy-Gigi, E., Richter-Levin, G., Korem, N., Shamay-Tsoory, S.G., 2016. The role of empathy in the neural responses to observed human social touch. Cogn. Affect. Behav. Neurosci.

Perry, A., Bentin, S., Bartal, I.B.-A., Lamm, C., Decety, J., 2010. "Feeling" the pain of those who are different from us: Modulation of EEG in the mu/alpha range. Cogn. Affect. Behav. Neurosci. 10, 493-504.

Pihko, E., Nevalainen, P., Stephen, J., Okada, Y., Lauronen, L., 2009. Maturation of somatosensory cortical processing from birth to adulthood revealed by magnetoencephalography. Clin. Neurophysiol. 120, 1552-1561.

Riečanský, I., Paul, N., Kölble, S., Stieger, S., Lamm, C., 2015. Beta oscillations reveal ethnicity ingroup bias in sensorimotor resonance to pain of others. Soc. Cogn. Affect. Neurosci. 10, 893-901.

Posner, M.I., 2008. Measuring alertness. Ann. N. Y. Acad. Sci. 1129, 193-199.

Rihs, T.A., Michel, C.M., Thut, G., 2009. A bias for posterior $\alpha$-band power suppression versus enhancement during shifting versus maintenance of spatial attention. Neuroimage 44, 190-199. 
Ritter, P., Moosmann, M., Villringer, A., 2009. Rolandic alpha and beta EEG rhythms' strengths are inversely related to fMRI-BOLD signal in primary somatosensory and motor cortex. Hum. Brain Mapp. 30, 1168-1187.

Saby, J.N., Meltzoff, A.N., Marshall, P.J., 2015. Neural body maps in human infants: Somatotopic responses to tactile stimulation in 7-month-olds. Neuroimage 118, $74-$ 78.

Sadaghiani, S., Kleinschmidt, A., 2016. Brain Networks and / -Oscillations: Structural and Functional Foundations of Cognitive Control. Trends Cogn. Sci. xx, 1-13.

Sauseng, P., Klimesch, W., Stadler, W., Schabus, M., Doppelmayr, M., Hanslmayr, S., Gruber, W.R., Birbaumer, N., 2005. A shift of visual spatial attention is selectively associated with human EEG alpha activity. Eur J Neurosci 22, 2917-2926.

Schaefer, M., Xu, B., Flor, H., Cohen, L.G., 2009. Effects of different viewing perspectives on somatosensory activations during observation of touch. Hum. Brain Mapp. 30, 2722-2730.

Schubert, R., Haufe, S., Blankenburg, F., Villringer, A., Curio, G., 2009. Now You'll Feel It, Now You Won't: EEG Rhythms Predict the Effectiveness of Perceptual Masking. Journal of cognitive neuroscience. Dec;21(12):2407-19.

Singer, T., Seymour, B., Doherty, J.O., Kaube, H., Dolan, R.J., Frith, C.D., 2004. Empathy for Pain Involves the Affective but not Sensory Components of Pain. Science (80-. ). 303, 1157-62.

Tecce, J.J., 1972. Contingent negative variation (CNV) and psychological processes in man. Psychol. Bull. 77, 73-108. 
Tecce, J.J., Scheff, N.M., 1969. Attention Reduction and Suppressed Direct-Current Potentials in the Human Brain. Science (3877 ). 164, 331-333.

Thut, G., Nietzel, A., Brandt, S.A., Pascual-Leone, A.,2006. Band Electroencephalographic Activity over Occipital Cortex Indexes Visuospatial Attention Bias and Predicts Visual Target Detection. The Journal of Neuroscience.13;26(37):9494-502.

Van Boxtel, G., Bocker, K.B.E., 2004. cortical measures of anticipation. J. Psychophysiol. 18, 130-139.

van Dijk, H., Schoffelen, J.-M., Oostenveld, R., Jensen, O., 2008. Prestimulus oscillatory activity in the alpha band predicts visual discrimination ability. J. Neurosci. 28, 1816-23.

van Rijn, H., Kononowicz, T.W., Meck, W.H., Ng, K.K., Penney, T.B., 2011. Contingent negative variation and its relation to time estimation: a theoretical evaluation. Front. Integr. Neurosci. 5, 1-5.

Walter, W.G., Cooper, R., Aldridge, V. J., McCallum, W.C., Winter, A.L., 1964. Contingent negative variation: an electric sign of sensori-motor association and expectancy in human brain. Nature. 203:380-384

Whitmarsh, S., Nieuwenhuis, I.L.C., Barendregt, H.P., Jensen, O., 2011. Sensorimotor Alpha Activity is Modulated in Response to the Observation of Pain in Others. Front. Hum. Neurosci. 5, 91.

Worden, M.S., Foxe, J.J., Wang, N., Simpson, G. V, 2000. Anticipatory biasing of 
visuospatial attention indexed by retinotopically specific-band electroencephalography increases over occipital cortex. J Neurosci,20(RC63), 1-6.

Yang, C.Y., Decety, J., Lee, S., Chen, C., Cheng, Y., 2009. Gender differences in the mu rhythm during empathy for pain: An electroencephalographic study. Brain Res. $1251,176-184$.

Zaki, J., Wager, T.D., Singer, T., Keysers, C., Gazzola, V., 2016. The Anatomy of Suffering: Understanding the Relationship between Nociceptive and Empathic Pain. Trends Cogn. Sci. 20, 249-259.

\section{List of Figures}

Figure 1. Alpha band $(8-14 \mathrm{~Hz})$ ERSP for the three conditions (self/other/nobody) during the anticipatory period, shown for the right hand (A) and left hand (B) separately. Within 
each section, time-frequency plots (lower panels) are shown for contralateral central electrodes in each condition. Topoplots (upper panels) show mean alpha ERSP during the window of $-1000 \mathrm{~ms}$ to $-500 \mathrm{~ms}$ before tactile stimuli were delivered (boxed area on time-frequency plots). Electrodes that show significant differences between the three conditions are indicated $(\mathrm{C})$.

Figure 2. Mean mu rhythm $(8-14 \mathrm{~Hz}) \mathrm{ERSP}$ at $\mathrm{C} 3$ and $\mathrm{C} 4$ from the onset of the visual cue $(-1500 \mathrm{~ms})$ to $500 \mathrm{~ms}$ post-stimulus.

Figure 3. Mean ERSP for mu rhythm $(8-14 \mathrm{~Hz})$ power in each condition during the window of $-1000 \mathrm{~ms}$ to $-500 \mathrm{~ms}$ prior to the onset of tactile stimuli. Negative values reflect a reduction in mu power (desynchronization) relative to a $500 \mathrm{~ms}$ pre-cue baseline, which was only observed in the "self" condition. When stimuli were delivered to the right hand (left panel), greater mu desynchronization was found at C3 than at C4.

Figure 4. The topographic distribution of anticipatory ERP (i.e., CNV) amplitude across the three conditions during the window of $-700 \mathrm{~ms}$ to $-500 \mathrm{~ms}$ (right panel). The left panel illustrates electrodes showing significant differences between the conditions $(p<0.01$, light blue; $p<0.001$, dark blue).

Figure 5. Mean ERP waveforms at electrodes C3 and C4 for each condition. Shadows surrounding each line represent standard errors. 
Figure 6. Mean CNV amplitude for each condition at electrodes C3 and C4. Mean amplitude was computed from a $800 \mathrm{~ms}$ window from $-900 \mathrm{~ms}$ to $-100 \mathrm{~ms}$ for each participant.

Figure 7. An example of one trial sequence. A trial was initiated by a fixation cross followed by a visual cue and then a target tactile stimulus. Three kinds of cues were presented: 1) a circle, indicating the stimulus would be delivered to the participant; 2) a square, indicating the stimulus would be delivered to the other person; 3) a triangle meant that a stimulus would occur but it would not be delivered to either person. The tactile stimulation was followed 500 ms later by a response prompt. 


\section{Figurestimulation to right hand}

Nobody -1000-500ms a a-14tz

A
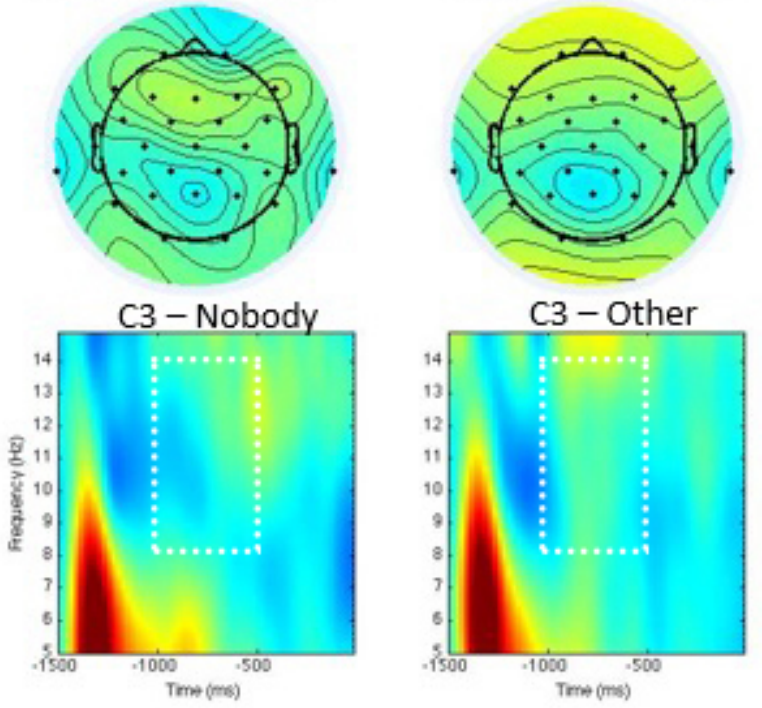

Stimulation to left hand

Nobody $-1000-500 \mathrm{ma}$ \& $8-14 \mathrm{~Hz}$
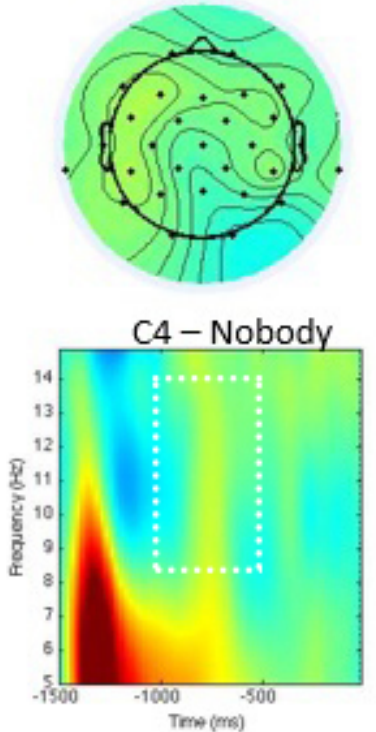

$\operatorname{Tm}(\mathrm{m} s)$

Other $-1000-500 \mathrm{~ms} s .8-14 \mathrm{~Hz}$
Other -1000-500ms a 8-14te
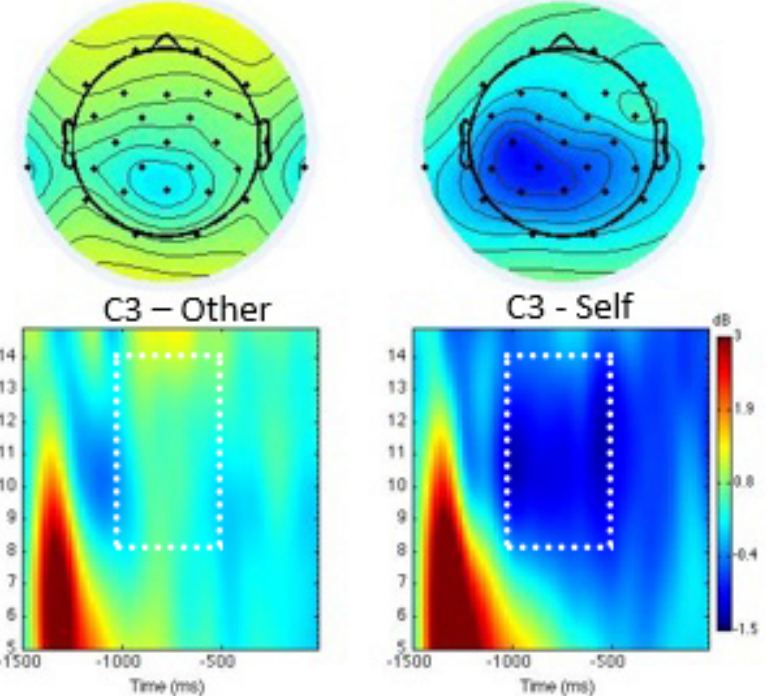

Trme (mins)
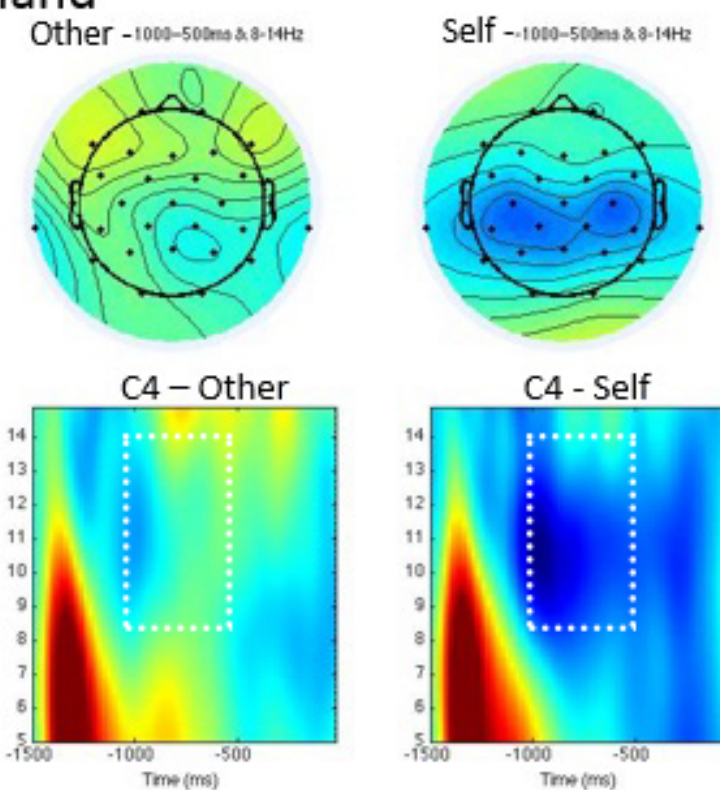

Self - 1000-500ms the 14 te
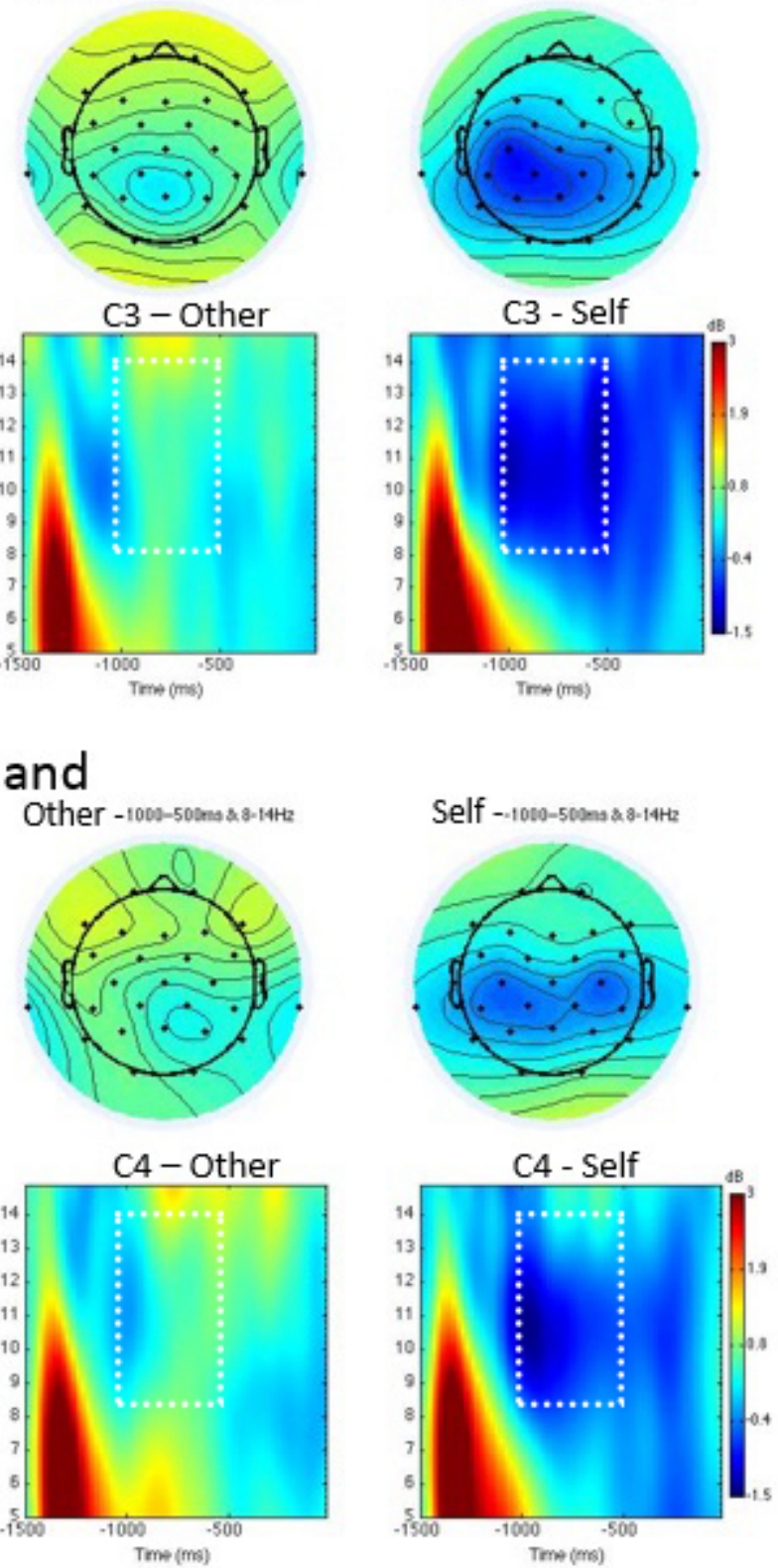

C

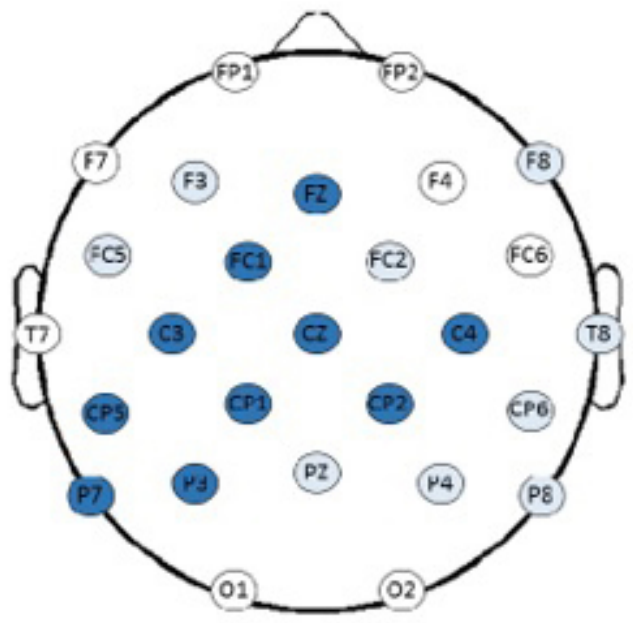

$\mathrm{p}<0.05 \quad \mathrm{p}<0.01$

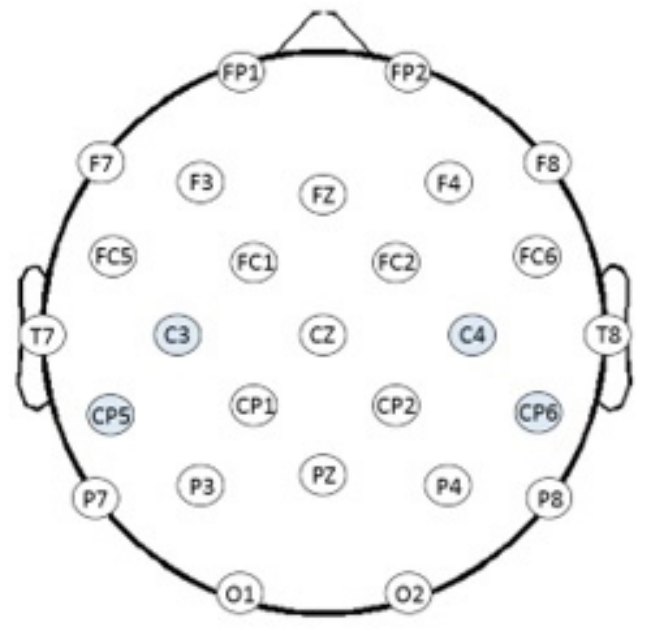



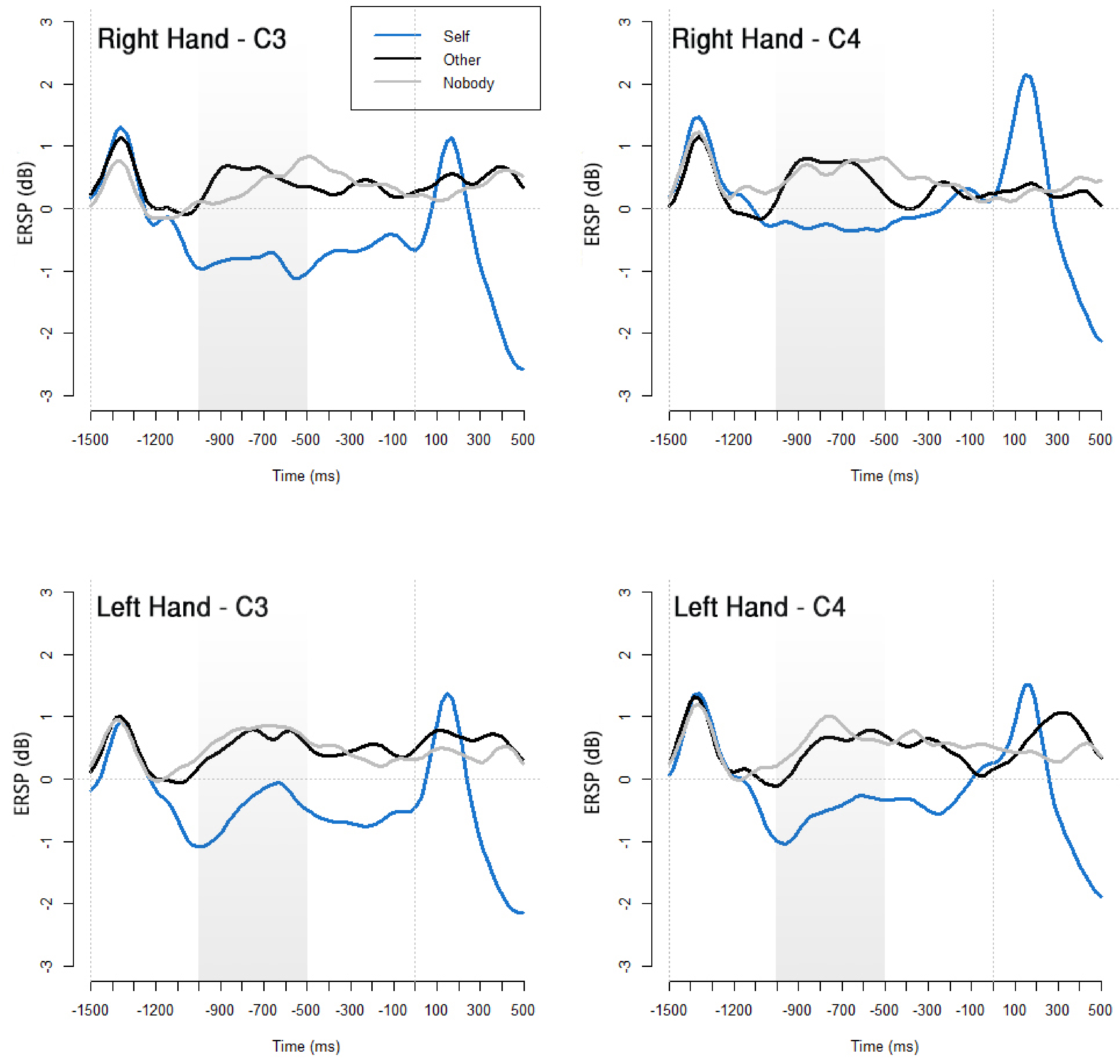
Left Hand

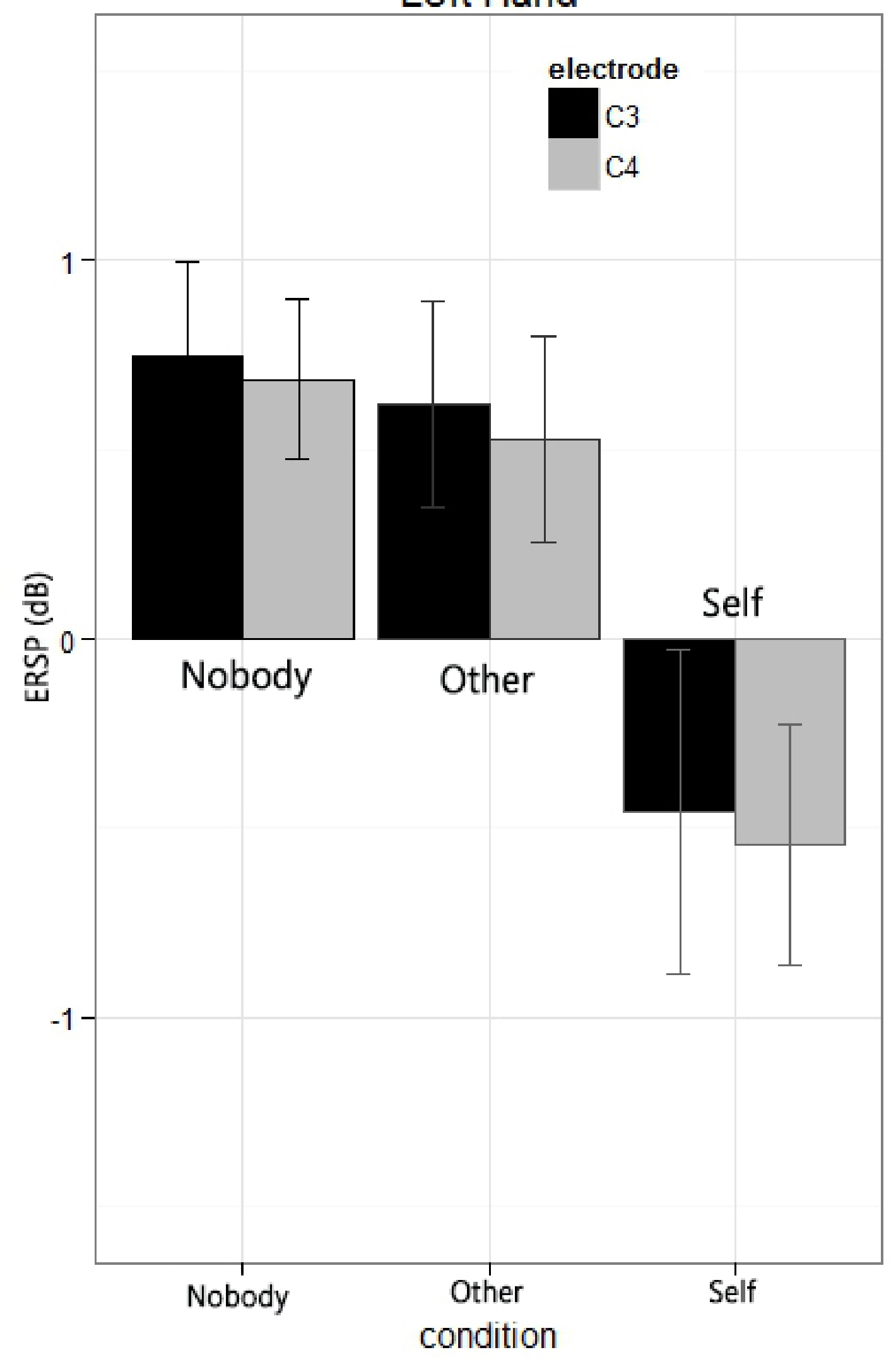

Right Hand

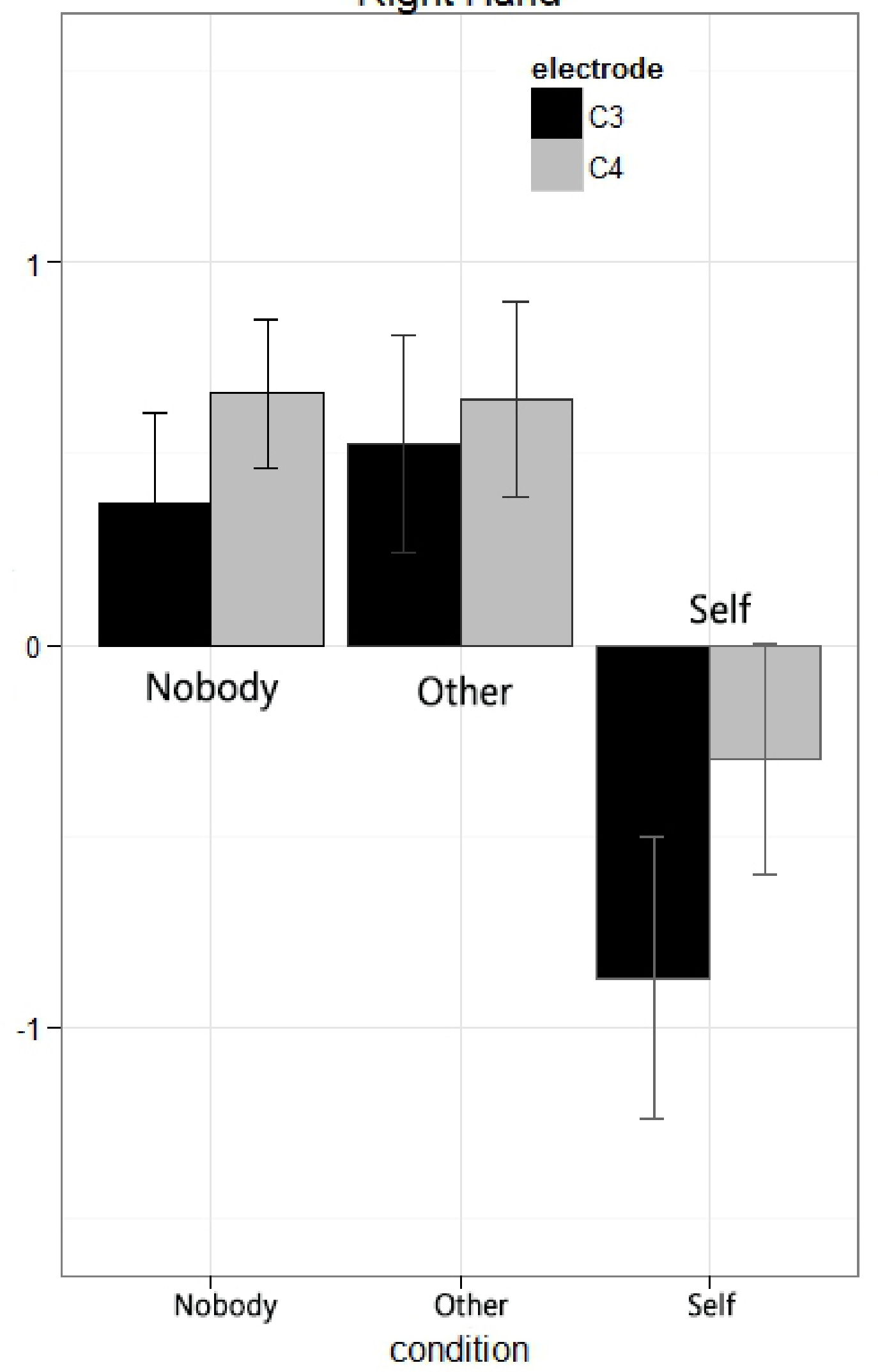



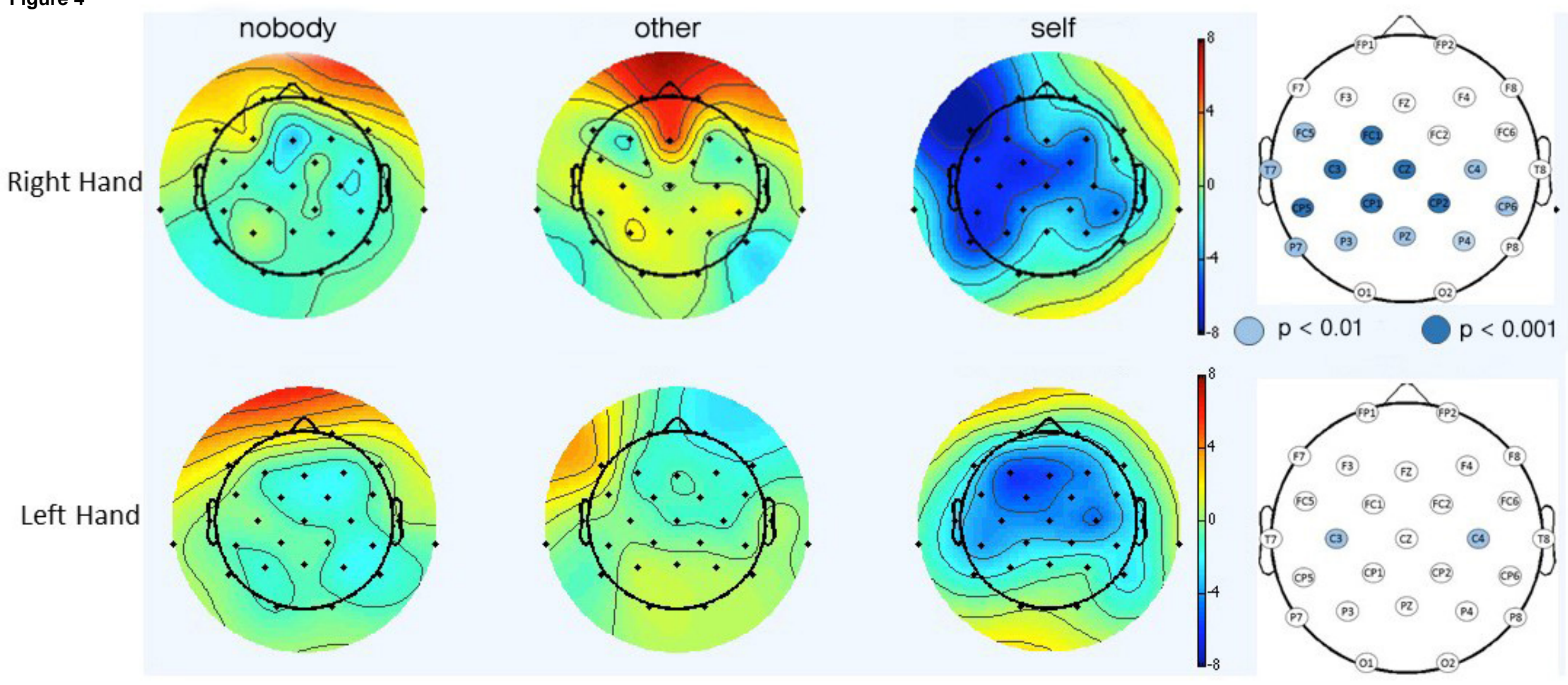

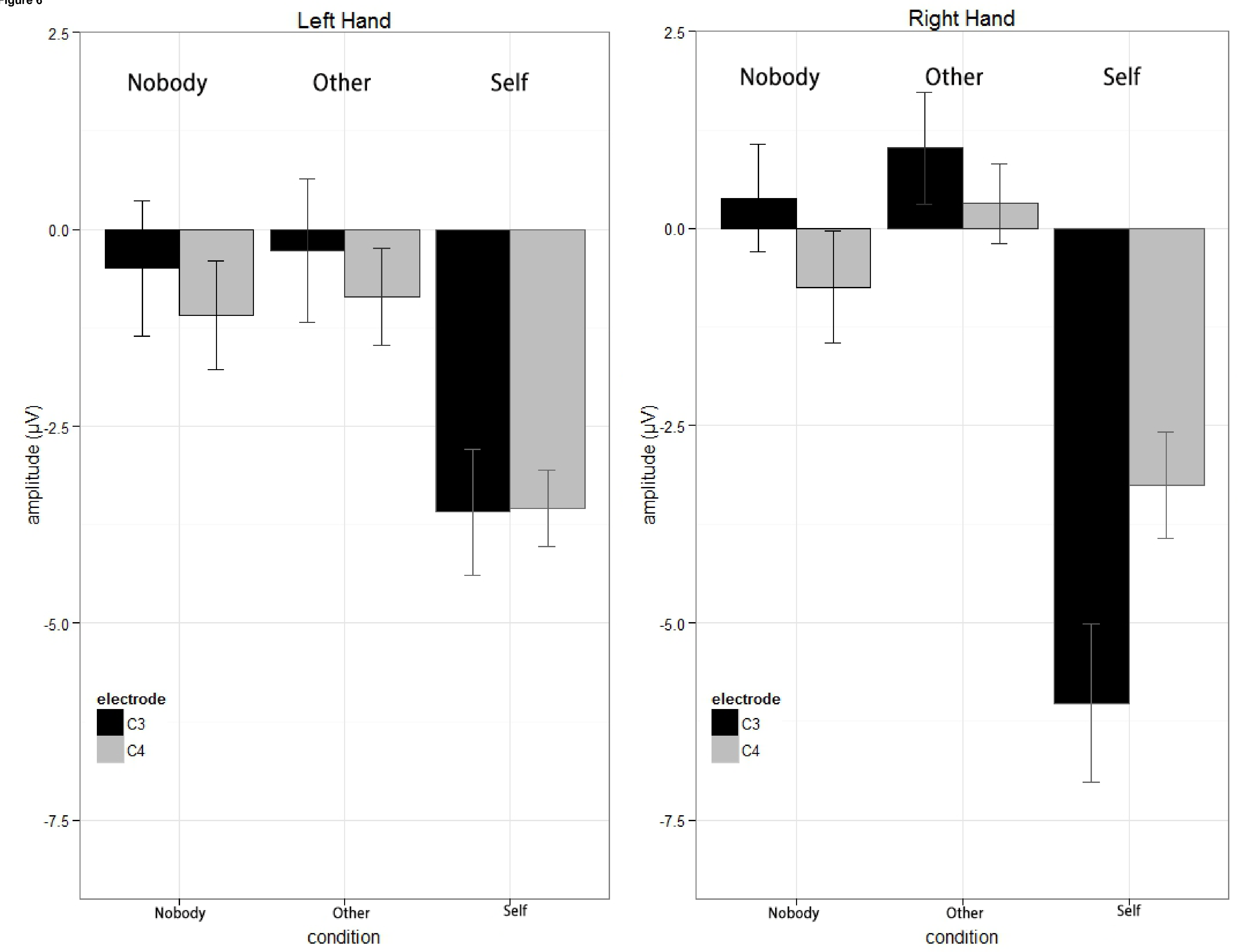

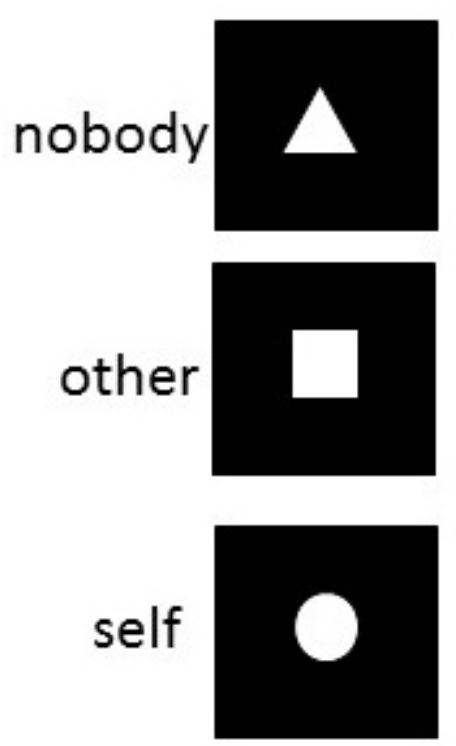

Fixation

$-3500 \mathrm{~ms}$
Cue

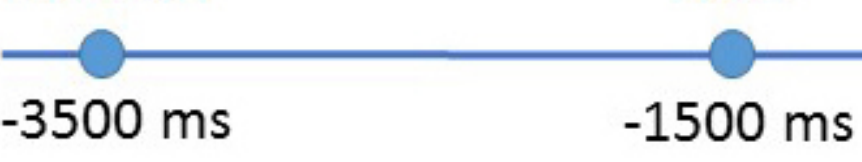

Long (5 pulses)

Short (3 pulses)

Tactile

stimuli

$0 \mathrm{~ms}$
Respond

Response

$500 \mathrm{~ms}$

$2500 \mathrm{~ms}$ 